\title{
Special Issue on Kardar-Parisi-Zhang Growth Dynamics
}

The 1986 contribution of Kardar, Parisi, and Zhang marks the then renewed interest in the statistical mechanics of non-equilibrium processes. They focused on the dynamics of growing interfaces resulting from the contact between a stable and metastable bulk phase, in spirit similar to an Eden growth process. In 2000 Johansson obtained an exact solution for the universal probability density of the random height of the growing interface above one point of the reference line. His method relies on a totally unexpected connection to the equilibrium statistical mechanics of line ensembles, in particular to Dyson's Brownian motion and the related Tracy-Widom distribution for the edge statistics of eigenvalues of random matrices. The predictions based on his and further exact solutions are beautifully confirmed in the 2010 turbulent liquid crystal experiments by Takeuchi and Sano.

Our special issue provides a momentary glimpse of current research activities on KPZ growth mostly in one dimension. We may point out that the introduction of Halpin-Healy and Takeuchi supplies a historical perspective. Exact solutions for the continuum KPZ equation are reviewed in the article of Quastel and Spohn. ${ }^{1}$

Herbert Spohn

Special Issue Editor

1 The article "Determinantal structures in the O'Connell-Yor directed random polymer model" by Takashi Imamura and Tomohiro Sasamoto is also a part of the KPZ special issue and it will be published in a later issue of Journal of Statistical Physics. 